\title{
«Les gens sont au courant et pourtant ils restent tout simplement indifférents!»
}

\begin{abstract}
Le récit autobiographique «Fleur du Désert» («Desert Flower») est un puissant et émouvant plaidoyer contre la mutilation des génitaux féminins. Son auteur, la somalienne Waris Dirie, décrit son parcours de petite fille d'une famille nomade qu'elle fuit pour échapper à un mariage forcé, et qui se retrouve quelques années plus tard topmodèle internationale. La version filmée du livre a connu un grand succès en Suisse alémanique. Le 17 mars prochain, il sera sur les écrans de la Suisse romande.* Dans I'interview Waris Dirie raconte la genèse du film et parle de son combat.
\end{abstract}

Interview et commentaires: Elke Eich

* Sortie du DVD en Suisse romande: automne 2010

** Nous insistons sur le fait que cette pratique d'oppression de femmes n'est pas d'origine islamique. Jusqu'en 1937 même en Europe et aux États Unis, elle était appliquée occasionnellement - par ex. pour soit-disant «traiter» des femmes «hystériques» ou nymphomanes.
1 Concernant les aspects de la prévention médicale de MGF (Mutilation Génitale Féminine) voir le travail qui est sorti en 2009 Jäger F, Hohlfeld P. Comment agir concrètement contre l'excision des filles en Suisse. Forum Med Suisse. 2009; 9(26-27):473-8. Online: www.smf.ch $\rightarrow$ Archives $\rightarrow$ $2009 \rightarrow 26 / 27$

2 Sources: entretiens de l'auteur avec C. Neuhaus et M. Killias. Voir aussi édition online du Journal «Sonntag» du 20.06.2009, http://www.sonntagonline.ch/ index.php?show=news\&type $=$ nachrichten\&id=384 [visité le 14.01.2010].
C'est dans des circonstances particulièrement tendues que le film «Fleur du Désert» est sorti en octobre dernier dans les salles de cinéma helvétiques. Son sujet principal, la mutilation génitale féminine (MGF) [1]), était à ce moment même un «sujet brûlant» en Suisse. En effet, un projet de loi prévoyait en été de mettre les pratiques de la MGF au même niveau qu'un piercing, permettant ainsi de la légaliser pour des femmes majeures et soulevant une tempête de critiques évoquées dans la consultation. Pour Martin Killias, professeur de droit pénal à Zurich, ce projet de loi est «choquant», et peut encourager un «tourisme pour des mutilations génitales». Le Directeur de la Justice à Berne, Christoph Neuhaus, a également pris une position ferme contre une telle «tolérance absolument fausse» [2]. Dans l'entretien avec l'auteur, il estime que la priorité doit avant tout être la protection des femmes qui risquent de subir une grande pression sociale, soit dans leur milieu de migrants ou soit bien dans leur pays natal. ${ }^{* *}$ Le débat n'est pas tranché et le Conseil national a décidé de prolonger, jusqu'à l'été 2011, les travaux de la commission chargée de la mise au point de ce projet. Quoi qu'il en soit, le film «Fleur du Désert» apporte à ce débat le témoignage poignant et convaincant d'une victime de ces pratiques ignobles.

\section{Rompre avec un tabou}

En effet, Waris Dirie est la première femme africaine qui a eu autant de retentissement pour avoir brisé pu-

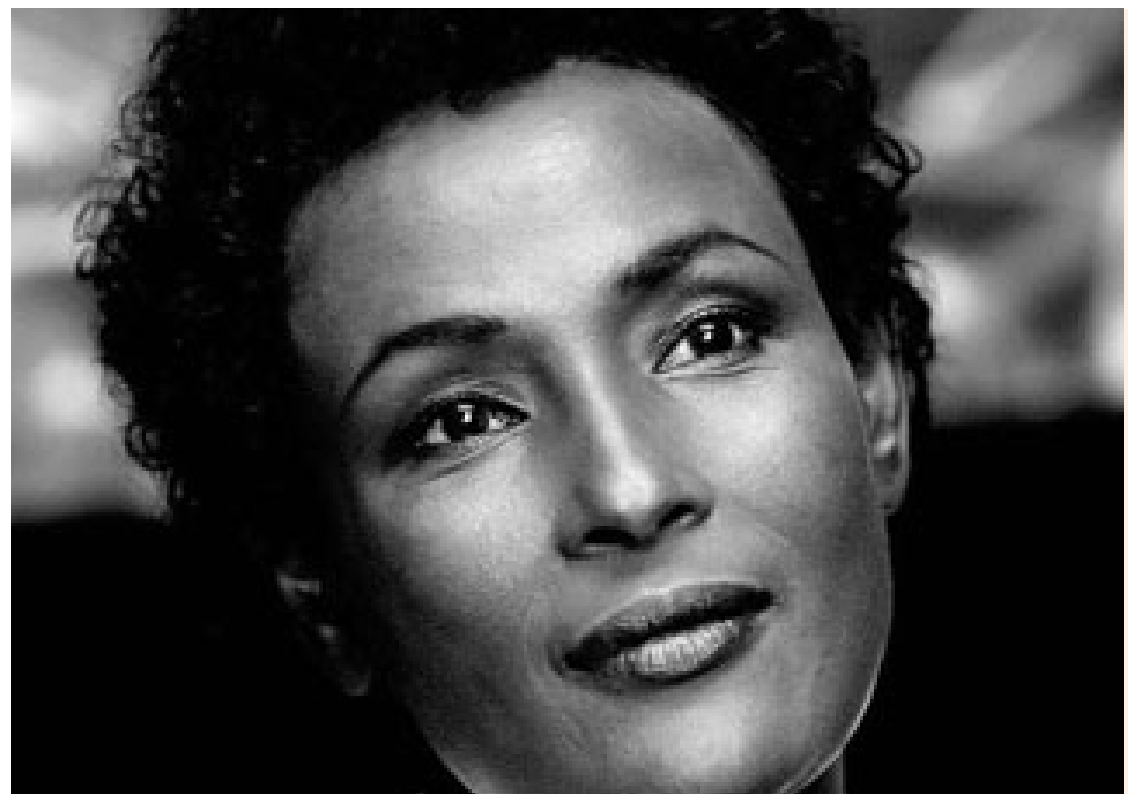

La mission de Waris Dirie, c'est la lutte contre la mutilation genitale féminine «Ma voix et mon visage maintenant sont connus exactement pour cela», dit l'ancienne top-modèle dans l'interview. 
bliquement et en toute conscience le grand tabou qui représente les pratiques de la MGF, d'abord dans des médias puis dans un discours légendaire prononcé devant l'ONU. Elle l'a fait avec toute la force de son caractère bien trempé et la légitimité de l'épreuve personnelle. Radicale, elle l'est dans sa dénonciation de traditions barbares; pugnace, elle l'est avec ceux qu'elle voulait et veut toujours informer et sensibiliser; courageuse, elle l'est avec son outing personnel.
C'est grâce à l'ancien mannequin vedette, auteur de quatre livres autobiographiques (le cinquième sortira ce printemps) et la sortie de son premier best seller «Fleur du Désert», que le débat sur la MGF a commencé à toucher à un large public, il y a plus de 10 ans.

Lors de l'entretien, cette complexe à la silhouette frêle, rayonnait de vitalité et de force de conviction. Toutes ces années de combat ont forgé son tempérament et sa manière d'être - sans concession.

\section{Interview avec Waris Dirie}

«Fleur du Désert»: captivant, touchant, choquant et pourtant très divertissant! Le producteur allemand Peter Herrmann, ethnologue de formation a une expérience du terrain africain. Lauréat d'un Oscar en 2003 pour «Nulle part en Afrique», il avait eu du flair pour le potentiel que ce récit de cette tranche décisive de la vie de Waris Dirie avait pour le cinéma. Pour la production de «Fleur du Désert» il a reçu le Prix bavarois du cinéma 2009.

Peu de gens - femmes ou hommes, peu importe - pourraient être insensible au film de la Germano-Américaine Sherry Hormann. L'aspect «cendrillon» de I'histoire fascinante de I'ancien top mannequin garantit un plein d'émotions. L'analphabète nomade fuit un mariage forcé qui la menace. Finalement elle arrive, passant par Mogadiscio, à Londres, où elle travaille comme servante de la famille de l'ambassadeur somalien, des parents éloignés. Pendant des années elle vit recluse, coupée du monde extérieur.

Suite au départ de la famille du diplomate, Waris (Liya Kebede) ne peut plus compter que sur elle-même. Elle se débrouille dans le tohu-bohu inconnu de Londres, sans passeport et avec quelques mots d'Anglais seulement, mais accompagnée de l'amitié de la vendeuse Marilyn (Sally Hawkins). Celle-ci, aussi «farfelue» que blanche, a été inventée pour le film, comme personnage miroir pour les occidentaux. Grâce à sa beauté exotique, la somalienne entre dans le monde du glamour et une carrière internationale comme mannequin s'ouvre pour elle. Le spectateur se réjouit du bonheur de Waris dont la vie maintenant prend de la vitesse, suivant le rythme de ce milieu agité et superficiel.

Le scénario du film fait du choc des cultures entre trois mondes - l'Afrique, I'occident et le glamour - un sujet de réflexion des aspects clé de la vie captivante de Waris Dirie, nourrie de ses racines africaines et poussée en avant de son grand désir d'affirmation de soi. L'intimité qui s'intensifie entre Waris et Marilyn est le catalyseur du sujet essentiel du film: la mutilation des génitaux féminins (MGF).

Au sommet de sa gloire, excédée par la vague d'articles sur sa carrière étonnante, présentée comme un conte de fées, Waris Dirie dévoile son vrai destin, celui d'une femme qui a souffert de l'infibulation, la pire des façons de la MGF. Et depuis son outing elle n'arrête pas de lutter contre ces pratiques barbares.

Le film de Hormann, inspiré du best seller de la somalienne résidant en Europe, raconte I'histoire avec une touchante profondeur, enveloppé dans le soufflé d'une comédie britannique pleine d'éléments divertissants. Et, dans son style particulier, «Fleur du Désert» introduit le sujet dur de la MGF d'une manière abordable qui se prête aussi bien à un usage pédagogique (écoles, ONG etc.) qu'à a une sensibilisation des décideurs politiques et juridiques. Il y a à faire: Plus de 8000 petites filles par jour sont victimes dans le monde entier, y compris dans les villes du «premier» et du «deuxième» monde (rien qu'à New York 40000 fois par an!).
Elke Eich: Madame Dirie, qu'est-ce qui vous pousse dans la vie?

L'émotion, des sentiments, des sensations... Tout tourne autour de ça. Pour moi, il n'y a que le brûlant ou le glacial!

Comment s'est déroulée votre première rencontre avec Sherry Hormann, réalisatrice et scénariste du film?

Quand Sherry et moi étions assises ensemble, ce fut un fort sentiment de détente, comme si je connaissais cette femme depuis toujours. Et le fait qu'elle soit une femme n'avait aucune importance.

Vous avez été donc tout de suite enthousiasmée par elle? En fait j'ai pensé: «une Blanche! Qui ne connaît rien à l'Afrique. Rien sur ce thème; qui n'a aucune idée de la vie dans le désert et ne connaît rien de comparable». J'avais en tête beaucoup de questions troublantes sur ce qu'elle voulait faire de mon livre.

L'avez-vous confrontée avec vos doutes?

Je lui ai dit: «Femme, parlons sérieusement: Tu le veux vraiment? Tu es sûre que tu veux rentrer là dedans? Comment veux-tu aborder cela? Et quel est ton but?» Je devais vraiment la sentir, la sonder et l'évaluer.

\section{Comment a-t-elle réagi?}

Elle a dit: «C'est simple, Waris. Il y a une raison pour laquelle je voudrais faire ce film. Il s'agit de la vie d'une femme. Rien de plus.».

Et cela a suffi pour obtenir votre accord et votre capital de confiance?

Je lui ai demandé tout simplement sa promesse de bien capter le message central de mon livre. Ce message qui est pour moi la seule raison de l'avoir écrit. Je lui ai dit: «Si tu captes bien ça, si le focus principal du film est de toucher les cœurs des gens, là je suis d'accord.» 
En clair, il s'agissait de quoi?

De la mutilation des génitaux féminins bien sûr. Elle devait forcement être présente dans le film, absolument crue, et aussi réaliste que possible, pour dilater les cours et les conquérir. Je voulais être sûre que les gens comprennent comment cela se passe, que c'est une véritable réalité. Et qu'il s'agit ici d'une guerre massive silencieuse contre les femmes, une guerre qui se déroule dans la clandestinité!

\section{«Pour moi, le sujet de la mutilation des génitaux féminins était la seule raison de faire ce film. Ce film devait capter ça parfaitement!»}

La scène de la mutilation n'est montrée que vers la fin du film dans un flash-back. Était-il indispensable de provoquer ce choc pour bousculer les gens?

Je me disais: j'ai déjà écrit et parlé de tout cela, j'ai sorti tout ce que j'ai vécu et tout ce que je sais sur ça. De sorte qu'effectivement le monde entier est au courant, vraiment tout le monde. Et alors? Les gens sont au courant et pourtant ils restent tout simplement indifférents. Ils attendent quoi, les politiciens, la justice, la police?! Même plus de dix ans après la publication de mon premier livre, cette pratique est toujours hors contrôle!

Vous m'avez dit que l'écriture d'un livre n'avait, au début, rien à voir avec vous, l'ancienne petite fille analphabète.

Mais j'étais obligée d'utiliser une arme. Et mon arme, c'est ma voix, ma propre expérience de la vie. Pour moi, le sujet de la mutilation des génitaux féminins était la seule raison de faire ce film. Ce film devait capter ça parfaitement.
Ma mutilation génitale ne représente pas toute ma vie qui est plutôt comme un volcan. J'ai dit aussi à Sherry: «Un tel destin, on ne peut supporter qu'avec de l'humour.» Mais après tout, ce film a été conçu pour toucher un large public, pour que les gens prennent enfin leur responsabilité et se demandent: «Qu'est-ce que je peux faire, moi?»

Pensez-vous, que suite à ce film, davantage de gens vont s'engager activement?

Oui, bien sûr. Nous pouvons faire des choses. Nous tous avons des opportunités et des responsabilités, et nous connaissons le problème. Le livre n'a pas entraîné l'action que je voulais. Mais le film pourrait devenir quelque chose de plus grand encore, quelque chose qui débouche sur des actes. Les actes sont plus forts que des mots. Donc, je veux que le monde soit choqué et je veux que les politiciens s'engagent parce que moi, en tant que personne seule, j'ai déjà fait assez.

Effectivement, vous avez lutté énormément dans votre vie et étiez souvent en danger. En plus, vous vous êtes exposée psychologiquement. Comment vous avez fait face à tout cela? Bon, maintenant je veux récupérer ma vie. Je veux m'éclater avec mes enfants. Je ne veux pas courir à droite à gauche comme un hamster sans tête, parlant toujours des mêmes trucs et les gens s'en f...! Cela me donne l'impression de prêcher dans le désert. J'ai le sentiment d'avoir donné ma voix à un comportement tellement cruel. Et ma voix et mon visage maintenant sont connus exactement pour cela. Et je déteste ça. En tout cas je suis loin d'en être heureuse.

Que pensez-vous de l'attitude de nombreuses Africaines qui ont souffert elles-mêmes de ces mutilations et qui continuent à les justifier comme étant des «prescriptions» traditionnelles?

\section{«Quand tu t'installes comme homme et tu dis: 〈' ai le pouvoir et toi, femme, tu ne peux rien faire de ta vie!) c'est inacceptable»}

Actuellement, et ceci dans toutes parties du monde, il y a encore plus de 8000 petites filles par jour qui sont victimes de différentes pratiques de la MGF, comment ce film peut contribuer, comme «arme» supplémentaire, à lutter pour changer cette situation?

Je voudrais vraiment que les spectateurs quittent la salle de cinéma avec le sentiment d'êtres humains transformés. Je veux changer votre vie, pour le mieux! Je veux vous apprendre quelque chose. Vous informer. Et je veux vous choquer.

Pourtant le film est aussi divertissant, plein d'humour. C'était aussi votre choix?
Elles préfèrent couvrir la pratique. Mais, après tout, tu veux lutter pour ta fille. Pour qu'elle ne doit pas vivre la même chose, qu'elle ne se retrouve pas dans la même situation que toi. C'est ce qu'on doit faire comme mère.

Pendant une longue période, dans les années cinquante et soixante, on a fait croire aux femmes en Occident que le lait de substitution était meilleur que le lait maternel, et donc beaucoup d'entre elles n'ont pas, à cette époque, donné le sein à leur bébé. Une grande partie de ces femmes ne pouvait guère supporter que les mères de nos jours aient le privilège de vivre cette expérience enrichissante. Le suc- 
cès d'une relation d'allaitement satisfaisante peut donc créer un sentiment de dépit chez celles qui ont raté pour toujours cette expérience. Boycotter les jeunes mères dans leur relation d'allaitement, par ex. en lançant des doutes, est un moyen fréquent de réagir à ce chagrin. Bien entendu la comparaison est relative compte tenu de la gravité de la MGF. Mais malgré cela, est-ce que vous reconnaissez un mécanisme de comportement identique parmi les femmes qui continuent de refouler les aspects négatifs de la MGF? C'est exactement ça. C'est un cercle. Comme chez ma propre mère. Elle voit comment je maîtrise ma vie et elle m'envie beaucoup. Elle ne le dirait jamais, mais je le sais. En cachette elle pense: «J'aimerais bien être comme toi.»

Qu'est-ce qui devrait arriver pour faire bouger les choses dans la bonne direction, en Afrique en particulier?

Il s'agit du renforcement du statut des femmes en Afrique. Je dis: «Donnez aux femmes la bonne éducation et tous les droits, et vous allez voir comme votre nation s'élève.»

Mais la plupart des hommes ne veulent pas abandonner leurs positions de pouvoir. Dans le monde occidental ils ne sont toujours pas ravis d'avoir à le faire, alors peut-on attendre des hommes africains qu'ils aillent dans votre sens?
Ils peuvent même garder leur pouvoir, tant qu'ils l'utilisent proprement. C'e n'est pas un problème. Mais quand tu t'installes comme homme et tu dis: «J'ai le pouvoir et toi, femme, tu ne peux rien faire de ta vie», ça c'est inacceptable.

Que pensez-vous de la performance de l'Éthiopienne Liya Kebede qui a incarné "votre» personnage? Après tout, elle a grandi dans une famille ouverte sur le monde et elle, au contraire de vous, était toujours soutenue pour faire ce qu'elle voulait de sa vie.

Oh, mon Dieu! Je ne veux pas mentir: au début je n'étais pas sûre, même si elle aussi est un mannequin comme je l'étais moi. Mais Liya a vraiment fait un travail d'excellence.

Pour vous, quels sont les moments les plus forts du film tourné en partie à Djibouti?

La scène la plus intense est celle de la mutilation. Elle m'a littéralement déchirée. C'est la scène la plus dure dans le film, mais elle est simplement capitale. Mais j'aime surtout les scènes africaines, les paysages africains, la Somalie magnifique. Là, mon cœur s'est ouvert!

\section{Note de lecture}

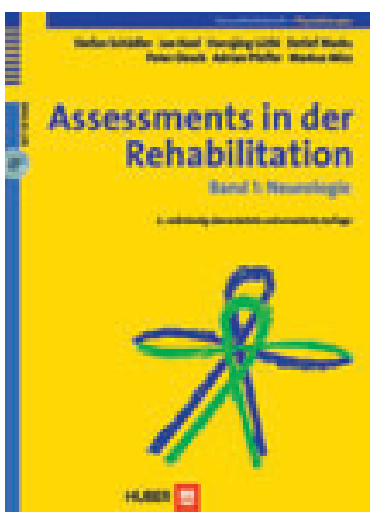

\section{Rehabilitation}

\section{Stefan Schädler et al.}

\section{Assessments in der Rehabilitation}

Band 1: Neurologie.

2. überarbeitete und erweiterte Auflage.

Bern: Hans Huber; 2009.

450 Seiten. Fr. 49.90.

ISBN 978-3-456-84630-9

Trotz erheblicher Fortschritte in der Behandlung neurologischer Krankheiten - als Beispiel dazu seien die Thrombolyse bei ischämischem Stroke oder die immer neuen medikamentösen und chirurgischen Behandlungsmethoden des Morbus Parkinson genannt - ist die grosse Mehrheit der Patienten für die Verbesserung ihrer Lebensqualität auf rehabilitatorische Massnahmen angewiesen. So hat in den letzten Jahren - auch im Zusammenhang mit der Verfügbarkeit immer neuer wissenschaftlicher Studien - die Bedeutung der Neurorehabilitation im Krankheitsmanagement stetig zugenommen. Bei gleichzeitig steigendem Kostenbewusstsein bzw. -druck im Gesundheitswesen müssen sich aber die in dieser Branche tätigen Spezialisten mit den Prinzipien der Evidence-Based-Medizin auseinandersetzen: Welche Massnahmen sind für welche Krankheitsbilder bzw. für welche Patienten am besten geeignet? Wie werden die Behandlungserfolge optimal gemessen?
Der erste Band des Buches «Assessments in der Rehabilitation», der sich auf über vierhundertfünfzig Seiten der Neurologie widmet, hilft als umfangreichste Sammlung von Messinstrumenten in deutscher Sprache, die obigen Fragen zu beantworten. Die Autoren dieses Werkes, das unter anderem von der Schweizerischen Gesellschaft für Neurorehabilitation unterstützt wurde, sind national sehr gut profilierte Physiotherapeuten, einige davon mit einem ausgeprägten Interesse für wissenschaftliche Fragen. Die überarbeitete und erweiterte 2 . Auflage beeindruckt durch die rationale und leserfreundliche Anordnung der unterschiedlichen Skalen. Hervorzuheben sind zudem die kritischen Kommentare der Autoren über deren Reliabilität, Validität und Responsivität; dabei werden Empfehlungen für die praktische Anwendung nicht vergessen. Als im Alltag taugliches Hilfsmittel ist ferner die beiliegende CD-ROM mit den Skalen in druckfähigem Format sehr nützlich.

Zusammenfassend handelt es sich hier um ein Buch, das in die Bibliothek einer jeden Rehabilitationsklinik gehört. Aber auch in Arztpraxen, die Physio- und/oder Ergotherapie anbieten, ist dieses wichtige Referenzwerk nicht fehl am Platz.

Dr. med. Fabio Baronti, Chefarzt und Med. Direktor, Klinik Bethesda, Neurorehabilitation, Parkinson-Zentrum, Epileptologie, Tschugg 